\title{
Medicina física e de reabilitação no tratamento da paralisia de Bell: qual a evidência?
}

Sofia Oliveira Vale, ${ }^{1}$ Sofia Marçalo, ${ }^{2}$ Cláudio Sousa Martins, ${ }^{3}$ Ana Catarina Machado ${ }^{4}$

\section{RESUMO}

Objetivo: Rever a evidência científica da medicina física e de reabilitação (MFR) na recuperação da paralisia de Bell (PB), na fase aguda ou crónica, em qualquer grau de severidade.

Fontes de dados: National Guideline Clearinghouse, Guidelines Finder, Canadian Medical Association Infobase, Cochrane Library, DARE e PubMed.

Métodos de revisão: Pesquisa de normas de orientação clínica (NOC), meta-análises (MA), revisões sistemáticas (RS) e estudos originais, em inglês, espanhol e português, publicados desde 2006, utilizando os termos MeSH peripheral facial paralysis, Bell palsy e rehabilitation. Para atribuição do nível de evidência dos estudos e da força de recomendação foi aplicada a escala Strength of Recommendation Taxonomy (SORT), da American Family Physician.

Resultados: Dos 127 artigos obtidos, sete preenchiam os critérios de inclusão: duas NOC, duas RS e três estudos originais. A Canadian Medical Association (2014) recomenda MFR na fase crónica, mas não na fase aguda, enquanto a National Guideline Clearinghouse (2013) não recomenda MFR, independentemente da fase. A RS de Ferreira e colaboradores (2015) revela maior recuperação motora com MFR do que com tratamento farmacológico isolado, sem discriminação da fase. A RS de Teixeira e colaboradores (2012) refere que há baixa evidência de que a MFR seja eficaz, tanto na fase aguda como na crónica. O estudo de coorte de Toffola e colaboradores (2012) conclui que doentes na fase aguda com axonotmese apresentam melhoria com MFR. O ensaio clínico aleatorizado e controlado (ECAC) de Monini e colaboradores (agosto de 2016) revela uma recuperação maior e mais rápida com MFR do que com tratamento farmacológico isolado na PB severa. O ECAC de Monini e colaboradores (dezembro de 2016) sugere a importância de combinar MFR com corticoterapia para um melhor resultado na recuperação da PB severa em todos os grupos etários.

Discussão: Os estudos têm pouca qualidade e são muito heterogéneos. Na fase aguda não permitem concluir se a recuperação dos doentes é espontânea ou se é devida/acelerada por MFR. Não há evidência consistente para a recomendação ou para a não recomendação de MFR na fase aguda ou crónica, pelo que não se pode atribuir uma força de recomendação. São necessários mais ECAC de boa qualidade.

Palavras-chave: Paralisia facial periférica; Paralisia de Bell; Reabilitação.

\section{INTRODUÇÃO}

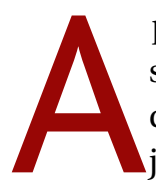
paralisia facial periférica (PFP) resulta da lesão neuronal periférica do nervo facial (VII) que pode ocorrer a qualquer nível do seu trajeto, do núcleo protuberancial à junção neuromuscular. ${ }^{1}$ Constitui a lesão periférica dos nervos cranianos mais comum. ${ }^{2}$ A PFP pode ser primária (idiopática ou paralisia de Bell [PB]), correspondendo a $75 \%$ dos casos, ou secundária a múltiplas causas, entre as quais diabetes mellitus, infeções víricas, tumores e traumatismo crânioencefálico. ${ }^{1}$

A PB tem uma incidência anual de 11,5-40,2 casos por 100.000 habitantes, podendo afetar até um em cada

\footnotetext{
1. Médica Especialista de Medicina Geral e Familiar na USF Nova Salus.

2. Médica Especialista de Medicina Geral e Familiar na USF S. Félix/Perosinho.

3. Médico Interno de Medicina Geral e Familiar na USF Arco do Prado.

4. Médica Interna de Medicina Geral e Familiar na USF Nova Salus.
} 
60 indivíduos ao longo da vida, com picos de incidência entre os 30-50 e os 60-70 anos..$^{3-4}$ Não existe predomínio por sexo ou hemiface afetada. ${ }^{1}$ A etiologia da PB é desconhecida, sendo admitidas como possíveis hipóteses uma infeção viral latente reativada (vírus herpes simplex ou varicella zoster), um mecanismo vascular ou de autoimunidade. ${ }^{1,3-5}$

A PB pode apresentar-se com diferentes graus de severidade, desde perda parcial a completa dos movimentos da hemiface afetada. ${ }^{4}$ Para objetivar o grau de lesão do nervo facial existem vários instrumentos, como a escala de House-Brackmann (HB) (Quadro I), uma das escalas mais utilizadas, e também a eletromiografia, que produz informações prognósticas quando realizada até 14 dias após o evento. ${ }^{1-6}$

A PB ocorre de forma aguda e pode evoluir para a cronicidade, não existindo uma definição consensual acerca da duração temporal da fase aguda e crónica. Além disso, alguns estudos nem as discriminam. ${ }^{1-5,7}$ Relativamente à recuperação, Peitersen ${ }^{8}$ demonstrou que cerca de $70 \%$ dos doentes apresenta uma recuperação espontânea completa, sendo em $85 \%$ nas três semanas após o evento e os restantes após três a cinco meses. Dada a elevada taxa de recuperação espontânea torna-se difícil estabelecer uma associação entre o tratamento e a recuperação, especialmente na fase aguda. ${ }^{5}$

A terapêutica com corticosteroides (prednisolona) parece ser a mais efetiva no tratamento da $\mathrm{PB}$, devendo ser iniciada nas primeiras 72 horas e é a única recomendada pelo Colégio Americano de Neurologia. ${ }^{4-5,7,9} \mathrm{~A}$ dose recomendada é de $60 \mathrm{mg}$ por dia durante cinco dias, seguida de 10mg por dia, numa duração total de tratamento de 10 dias. ${ }^{6}$ A utilização de corticosteroides na fase aguda da PB assenta no pressuposto da redução da inflamação e do edema do nervo facial.

Atualmente existem outras opções terapêuticas adjuvantes como os antivirais, a intervenção cirúrgica e a medicina física e de reabilitação (MFR). Contudo, a sua eficácia é controversa, quer no uso isolado quer em associação com a corticoterapia. Na MFR são vários os métodos e agentes físicos utilizados na terapia da $\mathrm{PB}$, como a reeducação neuromuscular funcional (associada ou não ao biofeedback com eletromiografia de superfície ou ao biofeedback do espelho), a mímica facial/exercícios faciais, a eletroestimulação, a massagem externa e a termoterapia. ${ }^{1,4-5}$

\section{QUADRO I. Escala de House-Brackmann}

Grau I: Normal

Função facial normal em todas as áreas

Grau II: Disfunção leve

Geral: leve fraqueza notável apenas à inspeção próxima; pode haver sincinésia muito discreta

No repouso: simetria e tónus normais

Ao movimento:

Testa: função boa a moderada

Olho: encerramento completo com mínimo esforço

Boca: leve assimetria

\section{Grau III: Disfunção moderada}

Geral: diferença óbvia, mas não desfigurante entre os dois

lados; sincinésia e/ou espasmo hemifacial notável, mas não graves

No repouso: simetria e tónus normais

Ao movimento:

Testa: movimento moderado a leve

Olho: encerramento completo com esforço

Boca: levemente fraca com o máximo esforço

Grau IV: Disfunção moderadamente importante

Geral: fraqueza óbvia e/ou assimetria desfigurante

No repouso: simetria e tónus normais

Ao movimento:

Testa: nenhum movimento

Olho: encerramento incompleto

Boca: assimetria com o máximo esforço

Grau V: Disfunção importante

Geral: apenas uma movimentação discretamente percetível

No repouso: assimetria

Ao movimento:

Testa: nenhum movimento

Olho: encerramento incompleto

Boca: movimento discreto

Grau VI: Paralisia total

Nenhum movimento

Legenda: Classificação da paralisia facial segundo a escala de House-Brackmann, da Academia Americana de Otorrinolaringologia.

Este trabalho tem como objetivo rever a evidência científica da MFR na recuperação da PB, na fase aguda ou crónica, em qualquer grau de severidade, definido por escalas validadas e/ou resultados de eletromiografia. 




Figura 1. Fluxograma do processo de seleção dos artigos.

Legenda: $\mathrm{ECAC}=$ Estudo clínico aleatorizado controlado; $\mathrm{NOC}=$ Norma de orientação clínica; RS = Revisão sistemática. neiro de 2006 e 31 de dezembro de 2017.

Os critérios utilizados para a inclusão dos artigos nesta revisão foram definidos segundo o modelo PICO: População doentes de qualqueridade com PFP Primária/PB na fase aguda e/ou crónica e com qualquer grau de severidade; Intervenção - qualquer modalidade de tratamento de MFR; Comparação - tratamento farmacológico isolado ou nenhum tipo de tratamento; Outcome - melhoria clínica avaliada por escalas validadas e/ou eletromiografia.

Foram excluídos os ECAC que comparam diferentes tratamentos de MFR.

Para estratificar o nível de evidência (NE) dos estudos e estabelecer a força de recomendação (FR) foi aplicada a Strength of Recommendation Taxonomy

\section{MÉTODOS}

Foi realizada uma pesquisa bibliográfica por dois autores da revisão, nas bases de dados National Guideline Clearinghouse, Guidelines Finder, Canadian Medical Association Infobase, Cochrane, DARE e PubMed. Foram pesquisadas normas de orientação clínica (NOC), meta-análises (MA), revisões sistemáticas (RS), ensaios clínicos aleatorizados e controlados (ECAC), ensaios clínicos não aleatorizados e estudos de coorte, utilizando os termos MeSH peripheral facial paralysis, Bell palsy e rehabilitation. Foram incluídos estudos em inglês, espanhol e português, publicados entre 1 de ja-
(SORT), da American Academy of Family Physicians. ${ }^{10-11}$

\section{RESULTADOS}

Dos 127 artigos obtidos da pesquisa, sete preencheram os critérios de inclusão: duas NOC, duas RS e três estudos originais (dois ECAC e um estudo de coorte). Foram eliminados 118 artigos após leitura do título e resumo, 15 dos quais por repetição e os restantes por não adequação ao tema ou por presença de critérios de exclusão. Dois ECAC foram excluídos após leitura comjá incluídas. A Figura 1 representa o fluxograma da seleção dos estudos. pleta dos artigos, uma vez que estavam inseridos em RS 


\begin{tabular}{|c|c|c|c|}
\hline Referência & Ano & Recomendações & FR \\
\hline $\begin{array}{l}\text { Canadian Medical } \\
\text { Association }^{3}\end{array}$ & 2014 & $\begin{array}{l}\text { Fase aguda, qualquer grau de severidade: } \\
\text { - Sem recomendação sobre exercícios de MFR } \\
\text { - Recomendam a não utilização de eletroestimulação } \\
\text { Fase crónica: } \\
\text { - Recomendação de exercícios de MFR }\end{array}$ & B \\
\hline $\begin{array}{l}\text { National Guideline } \\
\text { Clearinghouse }^{6}\end{array}$ & 2013 & $\begin{array}{l}\text { Não recomenda MFR com base em casos de séries: } \\
\text { - Baixo nível de confiança na evidência, por falhas significativas nos estudos analisados }\end{array}$ & B \\
\hline
\end{tabular}

Legenda: FR = Força de recomendação; MFR = Medicina física e de reabilitação.

\section{Normas de orientação clínica}

A Canadian Medical Association ${ }^{3}$ (2014) não recomenda os exercícios de MFR na fase aguda, qualquer que seja o grau de severidade da PB, baseando-se em três ECAC. Relativamente à eletroestimulação, na fase aguda é recomendada a sua não utilização, uma orientação baseada em quatro estudos (ECAC ou quasi-aleatorizados). Na fase crónica, esta NOC recomenda os exercícios de MFR nos pacientes com défice persistente, com base num estudo não aleatorizado (o grupo submetido a MFR teve uma melhoria significativa da função facial medida pela escala HB, com um RR [risco relativo] de 0,6 e um IC [intervalo de confiança] 95\% [0,1-1,1]) (Quadro II). As conclusões desta NOC baseiam-se em estudos de fraca qualidade, com risco de viés e resultados imprecisos e heterogéneos. Além disso, não define a duração da fase aguda e crónica (FR B).

A National Guideline Clearinghouse ${ }^{7}$ (2013) não recomenda MFR, independentemente da fase (Quadro II). Baseia-se em casos de séries com baixo nível de confiança na sua evidência por falhas significativas nos estudos incluídos, com balanço duvidoso entre benefícios e riscos, não discriminando também fase aguda de fase crónica (FR B).

\section{Revisões sistemáticas}

A RS de Teixeira e colaboradores, ${ }^{4}$ publicada em 2012 (Quadro III), inclui 12 ECAC ou quasi-aleatorizados, num total de 873 participantes. Os autores da presente revisão optaram apenas por incluir os únicos quatro estudos (364 participantes) que avaliaram a eficácia do tratamento de MFR (exercícios faciais ou eletroestimulação) face a um grupo controlo sem MFR. ${ }^{12-15}$ Esta
RS teve como objetivo primário analisar a recuperação incompleta seis meses após o evento. Teve como, objetivos secundários, analisar a presença de sincinésias motoras, contraturas, hipersincinésias, espasmos faciais ou síndroma de Bogorad, seis meses após o evento; analisar a recuperação incompleta após um ano; e analisar os efeitos adversos associados à intervenção. A maioria dos resultados não demonstrou diferença estatisticamente significativa entre os tratamentos. Apesar de não ter significado estatístico, a MFR quando aplicada durante duas semanas promoveu uma recuperação mais rápida nos casos mais severos (no grupo experimental recuperaram cinco dos nove doentes, comparando com o grupo controlo em que apenas três dos onze doentes recuperaram, com um $\mathrm{RR}=2,4$ e com IC95\% $[0,66-6,29]) .{ }^{12}$ Relativamente às sincinésias motoras, numa pequena amostra (grupo experimental $=4$ $[4,7 \%]$ versus grupo controlo $=12[20 \%]$ ) verificou-se uma redução após 12 semanas de exercícios faciais $(\mathrm{RR}=0,24, \mathrm{IC95 \%}[0,08-0,69]) .{ }^{13}$ Quanto à fase crónica (mais de nove meses após o evento), verificou-se que ao fim de um ano de MFR todos os participantes passaram de uma pontuação de IV na escala HB para uma pontuação de III $(\mathrm{RR}=2,40$, IC95\% $[8,76-32,04]) .{ }^{14}$ No que respeita à electroestimulação, não se verificou um benefício significativo quando comparada com a corticoterapia (recuperação completa seis meses após: grupo experimental $=10[12,98 \%]$ versus grupo controlo=11 [15,27\%], com RR=0,62, IC95\% $[0,34-1,15]) .{ }^{15}$ Não houve registo de efeitos adversos da MFR em nenhum dos estudos incluídos. Esta RS inclui estudos de baixa ou moderada qualidade, com populações heterogéneas e dados incompletos. Três estudos avaliam a eficácia de 


\begin{tabular}{|c|c|c|c|c|}
\hline Autor (ano) & Estudos incluídos & Intervenção & Conclusões & $\mathrm{NE}$ \\
\hline $\begin{array}{l}\text { Teixeira, et al. } \\
(2012)^{3}\end{array}$ & $\begin{array}{l}12 \text { ECAC ou quasi- } \\
\text {-randomizados }\end{array}$ & $\begin{array}{l}\text { - Quatro estudos avaliam eficácia } \\
\text { de tratamento de MFR: } \\
\text { - Beurskens, et al, }{ }^{12} \text { MFR vs sem } \\
\text { tratamento farmacológico } \\
\text { - Wen, et al, }{ }^{13} \text { e Barbara, et al, }{ }^{14} \\
\text { MFR associada a tratamento } \\
\text { farmacológico vs tratamento } \\
\text { farmacológico } \\
\text { - Flores, et al, }{ }^{15} \text { eletroestimulação } \\
\text { vs corticoterapia } \\
\text { - Restantes estudos: } \\
\text { - Três estudos avaliam } \\
\text { eletroestimulação associada a } \\
\text { MFR vs MFR } \\
\text { - Cinco estudos avaliam } \\
\text { tratamento de acupuntura }\end{array}$ & $\begin{array}{l}\text { - Exercícios faciais: } \\
\text { - Se realizados nos três meses iniciais } \\
\text { poderão prevenir sincinésias motoras } \\
\text { - Diminuem o tempo para recuperação } \\
\text { completa em casos mais severos } \\
\text { - Podem ser benéficos na fase crónica } \\
\text { - Eletroestimulação sem benefício vs } \\
\text { corticoterapia }\end{array}$ & 2 \\
\hline $\begin{array}{l}\text { Ferreira, et al. } \\
(2015)^{4}\end{array}$ & $\begin{array}{l}4 \text { ECAC ou quasi- } \\
\text {-randomizados }\end{array}$ & $\begin{array}{l}\text { - Nicastri, et al, }{ }^{16} \text { Barbara, et al, }{ }^{14} \\
\text { Penteado, et al, }{ }^{17} \text { tratamento } \\
\text { farmacológico associado a MFR } \\
\text { vs tratamento farmacológico } \\
\text { isolado } \\
\text { - Alakram, et al, }{ }^{18} \text { corticoterapia } \\
\text { associada a MFR vs corticoterapia } \\
\text { associada a MFR com } \\
\text { eletroestimulação }\end{array}$ & $\begin{array}{l}\text { - Maior recuperação motora que } \\
\text { tratamento farmacológico isolado } \\
\text { - Melhoria estatisticamente significativa } \\
\text { quando iniciada precocemente nos graus } \\
\text { severos de paralisia (HB V e VI) } \\
\text { - Eletroestimulação sem influência nos } \\
\text { resultados do tratamento }\end{array}$ & 2 \\
\hline
\end{tabular}

Legenda: $\mathrm{ECAC}=$ Estudo clínico aleatorizado controlado; MFR = Medicina física e de reabilitação; vs = versus.

MFR na fase aguda, mas definem de forma diferente a duração desta fase, com um máximo de 12 semanas. ${ }^{12-13,15}$ Apenas um estudo incluído avalia a eficácia de MFR na fase crónica (definida por uma duração superior a nove meses), tratando-se, no entanto, de um estudo de fraca qualidade. ${ }^{14}$ Tendo em conta as limitações apontadas nestes estudos atribui-se um NE 2.

A RS de Ferreira e colaboradores, ${ }^{5}$ publicada em 2015 (Quadro III), inclui quatro estudos ECAC ou quasi-aleatorizados, num total de 143 participantes com idades entre os 16 e 87 anos. Três estudos comparam a eficácia da MFR associada a tratamento farmacológico com o tratamento farmacológico isolado; o outro estudo inclui também eletroestimulação associada à MFR..$^{12,16-18}$ Esta RS teve como objetivo primário medir a recuperação muscular facial (parcial ou completa), definida pela escala HB nos graus I ou II; e, como objetivo secundário, medir os efeitos adversos da intervenção. Esta RS demonstrou maior recuperação motora com MFR associada a tratamento farmacológico do que com tratamento farmacológico isolado. Um dos estudos, considerado de boa qualidade, em que a MFR foi iniciada até 10 dias após o evento de $\mathrm{PB}$, demonstrou que o grupo experimental (MFR e corticoterapia) teve uma melhoria significativa na recuperação funcional $(p=0,044)$, quando comparado com o grupo controlo (corticoterapia isolada), nos graus severos de paralisia ( $\mathrm{HBV} \mathrm{eVI})$ após seis meses (Quadro III). ${ }^{16} \mathrm{~A}$ eletroestimulação associada a corticoterapia mostrou uma recuperação da função motora (grupo experimental=37\% e grupo controlo=30\%), embora não estatisticamente significativa $(p=0,36)$, permitindo concluir que a eletroestimulação 


\begin{tabular}{|c|c|c|c|c|c|}
\hline Autor (ano) & Tipo & Metodologia & Intervenção & Conclusões & NE \\
\hline $\begin{array}{l}\text { Monini, et al. } \\
\text { (agosto 2016) }^{19}\end{array}$ & ECAC & $\begin{array}{l}94 \text { Participantes (entre os } \\
16 \text { e } 90 \text { anos) } \\
\text { Critérios de inclusão: } \\
\text { - Admissão no SU } \\
\text { - Diagnóstico de PB severa } \\
\text { (HB graus IV e V) } \\
\text { Composição dos grupos de } \\
\text { estudo definida pela atitude } \\
\text { individual da equipa }\end{array}$ & $\begin{array}{l}\text { - Grupo controlo }(n=66) \\
\text { tratado com corticoterapia } \\
\text { - Grupo experimental } \\
(n=28) \text { tratado com } \\
\text { corticoterapia e MFR - } \\
\text { método de Kabat }\end{array}$ & $\begin{array}{l}\text { - Maior recuperação } \\
\text { motora e mais rápida com } \\
\text { MFR do que com } \\
\text { tratamento farmacológico } \\
\text { isolado }\end{array}$ & 1 \\
\hline $\begin{array}{l}\text { Monini, et al. } \\
\text { (dezembro } \\
2016)^{20}\end{array}$ & ECAC & $\begin{array}{l}104 \text { Participantes } \\
\text { Critérios de inclusão: } \\
\text { - Admissão no SU } \\
\text { - Diagnóstico de PB severa } \\
\text { (HB graus IV e V) } \\
\text { Composição dos grupos de } \\
\text { estudo definida de forma } \\
\text { aleatorizada } \\
\text { Distinção em cada grupo de } \\
\text { dois subgrupos: não idoso } \\
\text { ( } \leq 65 \text { anos) e idoso } \\
\text { (> } 65 \text { anos) }\end{array}$ & $\begin{array}{l}\text { - Grupo controlo ( } n=66 \text { ) } \\
\text { tratado com corticoterapia } \\
\text { ( } 44 \text { do subgrupo não idoso } \\
\text { e } 22 \text { do idoso) } \\
\text { - Grupo experimental } \\
\text { ( } n=38 \text { ) tratado com } \\
\text { corticoterapia e MFR - } \\
\text { método de Kabat ( } 28 \text { do } \\
\text { subgrupo não idoso e } 10 \\
\text { do idoso) }\end{array}$ & $\begin{array}{l}\text { - Maior recuperação } \\
\text { motora e mais rápida com } \\
\text { MFR do que com } \\
\text { corticoterapia em todos } \\
\text { os grupos etários } \\
\text { - Tratamento combinado } \\
\text { particularmente } \\
\text { importante nos doentes } \\
\text { mais idosos e com grau } \\
\text { mais severo de PB (HB V) }\end{array}$ & 1 \\
\hline $\begin{array}{l}\text { Toffola, et al. } \\
(2012)^{2}\end{array}$ & $\begin{array}{l}\text { Estudo de } \\
\text { coorte }\end{array}$ & $\begin{array}{l}102 \text { Casos ( } 12 \text { aos } 80 \text { anos) } \\
\text { Critérios de inclusão: } \\
\text { - Doentes em fase aguda } \\
\text { (< } 16 \text { dias) } \\
\text { - EMG 3-4 semanas após } \\
\text { evento } \\
\text { - Follow-up } 12 \text { meses } \\
\text { Classificação do grau de } \\
\text { severidade através da escala } \\
\text { de HB e de EMG }\end{array}$ & $\begin{array}{l}\text { - } 73 \text { Doentes com } \\
\text { axonotmese submetidos a } \\
\text { tratamento com MFR ( } 38 \\
\text { EMG-BFB e } 35 \\
\text { MIRROR-BFD) } \\
\text { - } 29 \text { Doentes com } \\
\text { neuropraxia não } \\
\text { submetidos a MFR }\end{array}$ & $\begin{array}{l}\text { - Recomenda tratamento } \\
\text { com MFR na axonotmese } \\
\text { - Não recomenda o } \\
\text { tratamento com MFR na } \\
\text { neuropraxia }\end{array}$ & 2 \\
\hline
\end{tabular}

Legenda: $\mathrm{ECAC}=$ Ensaio clínico aleatorizado controlado; $\mathrm{EMG}=$ Eletromiografia; $\mathrm{EMG}-\mathrm{BFB}=$ biofeedback por eletromiografia; $\mathrm{MIRROR}-\mathrm{BFD}=$ biofeedback do espelho; HB = House-Brackmann; MFR = Medicina física e de reabilitação; SU = Serviço de urgência.

não tem influência nos resultados do tratamento padrão da PB..$^{18}$ Nenhum estudo incluído reportou efeitos adversos. Esta RS inclui poucos estudos e a maioria deles tem evidência de baixa qualidade, excetuando um, considerado de boa qualidade (com base na escala PEDro). Além disso, os estudos instituem tratamentos de MFR em fases temporais diferentes, que variam entre os quinze dias e um ano. Desta forma, não é possível concluir se o tratamento combinado (MFR e corticote- rapia) é ou não benéfico na recuperação motora dos doentes com PB, pelo que se atribuiu um NE 2.

\section{Estudos originais \\ Estudo coorte}

O estudo de coorte de Toffola e colaboradores, ${ }^{2}$ de 2012 (Quadro IV), incluiu 102 participantes (entre os 12 e 80 anos) na fase aguda da PB (menos de 16 dias após o evento), submetidos a eletromiografia 3-4 semanas 
após o evento (permitindo distinguir neuropraxia de axonotmese) e com follow-up aos 12 meses. Este estudo utilizou a escala de HB (no início e 12 meses depois) e a eletromiografia (cerca de um mês após o evento) para classificação do grau de severidade. Dos participantes incluídos, 29 doentes tinham neuropraxia (bloqueio fisiológico transitório na contusão nervosa e com continuidade anatómica mantida) e não foram submetidos a qualquer tratamento de MFR. A axonotmese (rutura dos axónios, com manutenção da integridade da bainha) estava presente em 73 doentes, que foram submetidos a tratamento com MFR (38 doentes com biofeedback por eletromiografia e 35 doentes com biofeedback do espelho).

Houve uma melhoria clínica no final dos 12 meses em todos os participantes do estudo, mas sem significado estatístico $(p=0,596)$. Todos os doentes com neuropraxia apresentaram recuperação espontânea completa, incluindo os $37,9 \%$ que tinham paralisia total (HB VI). Quanto aos doentes com axonotmese, em 53,5\% dos casos com paralisia total, apenas $34 \%$ tiveram recuperação completa, avaliada pela escala HB. Uma das limitações deste estudo é o facto de ter sido realizado apenas em pacientes na fase aguda de PB. Atribuiu-se um NE 2.

\section{$\underline{\text { ECAC }}$}

O ECAC de Monini e colaboradores, ${ }^{19}$ de agosto de 2016 (Quadro IV), incluiu 94 participantes (entre os 16 e 90 anos) admitidos no serviço de urgência com diagnóstico de PB severa (HB graus IV e V). Os participantes foram divididos em dois grupos com base na atitude terapêutica: grupo controlo $(n=66)$ tratado com corticoterapia e grupo experimental $(n=28)$ tratado com corticoterapia e MFR - método de Kabat (facilitação neuromuscular propriocetiva da resposta voluntária do músculo lesado).

Ao comparar o grupo experimental com o grupo controlo, independentemente do grau inicial de PB, foram encontradas diferenças significativas $(p=0,010)$ naquilo que respeita à melhoria clínica. O significado estatístico relativamente às duas modalidades de tratamento (grupo experimental versus controlo) foi ainda maior quando analisados individualmente os graus IV $(p=0,000)$ eV $(p=0,009)$ da escala de HB.

No que respeita às especificidades da recuperação, os participantes do grupo experimental apresentaram uma probabilidade de melhoria, em três ou mais graus na escala de HB, cerca de 20 vezes superior, à apresentada pelos participantes do grupo controlo (Odds-Ratio $[\mathrm{OR}]=17,73$, IC95\% [5,72-54,98], $p<0,001)$. Quando analisados individualmente, os participantes do grupo experimental com HB IV apresentaram uma probabilidade de melhoria, em três graus na escala de HB, 65 vezes superior à do grupo controlo; enquanto todos os participantes do grupo experimental com HB V tiveram melhoria em três graus na escala de HB, quando comparados com o grupo controlo. Em suma, a maioria dos pacientes com $\mathrm{PB}$ grave (HB V) do grupo controlo alcançou o resultado máximo de HB III, enquanto os casos semelhantes do grupo experimental (submetidos ao método de Kabat) conseguiram obter uma função facial normal (HB I).

Em relação à velocidade média de recuperação, no grupo experimental foi metade da registada no grupo controlo (RR=2,19, IC95\% [1,37-3,51], $p=0,001$ ). Os pacientes do grupo experimental com HB IV tiveram tempos de recuperação sete vezes mais curtos, em relação aos pacientes do grupo controlo com graus semelhantes de PB (RR=7,92, IC95\% [3,68-17,04], $p<0,001)$. Já os pacientes do grupo experimental com HBV apresentaram um tempo de recuperação cerca de 50\% inferior aos pacientes do grupo controlo com o mesmo grau de PB (RR=1,80, IC95\% [0,65-5,01], $p=0,26)(64,6$ dias versus 117,1 dias).

A incidência de sincinésias após o tratamento foi de $7,1 \%$ no grupo experimental e de $12,1 \%$ no grupo controlo, sem diferença estatisticamente significativa ( $p=0,479)$.

Este estudo apresenta várias limitações. Primeiro, os grupos de estudo foram escolhidos com base na avaliação da equipa do serviço de urgência, que definiu aleatoriamente quais os doentes a serem sujeitos a terapêutica no domicílio com corticoterapia e quais os doentes a referenciar para a consulta para realização de MFR (método de Kabat). Segundo, incluiu apenas pacientes com PB severa (HB IV e V). Por último, não foi discriminada a fase da PB em que os doentes se encontravam, pois apesar de terem sido incluídos doentes que se deslocaram ao serviço de urgência (possivelmente em fase aguda), não está descrito quando é que estes iniciaram os sintomas. Atribuiu-se um NE 1. 
Outro ECAC, também de Monini e colaboradores, ${ }^{20}$ de dezembro de 2016 (Quadro IV), incluiu 104 participantes admitidos no serviço de urgência de um hospital terciário, com o diagnóstico de PB severa (HB graus IV e V). Os participantes foram divididos em dois grupos com base na atitude terapêutica: grupo controlo ( $n=66)$ tratado com corticoterapia e grupo experimental ( $n=38)$ tratado com corticoterapia e MFR - método de Kabat. Em ambos os grupos foram distinguidos dois subgrupos: um subgrupo com idade igual ou inferior a 65 anos de idade (subgrupo não idoso) [grupo controlo $(n=44)$ versus grupo experimental $(n=28)]$ e um subgrupo com idade superior a 65 anos de idade (subgrupo idoso) [grupo controlo ( $n=22)$ versus grupo experimental $(n=10)]$.

A análise de covariância da recuperação temporal, independentemente da idade, mostrou que o tempo de recuperação foi significativamente menor no grupo experimental $(p=0,004)$. Neste grupo, considerando os participantes com PB de HBV, quase $90 \%$ do subgrupo não idoso apresentou uma recuperação completa, enquanto esse resultado foi de apenas cerca de $50 \%$ no subgrupo idoso. Em relação aos participantes com HB IV não se verificou qualquer diferença de recuperação entre os dois subgrupos de idade, uma vez que a recuperação completa foi alcançada na totalidade dos participantes.

No que respeita ao tempo médio para a recuperação clínica no subgrupo não idoso, no grupo controlo a duração foi de 103,2 dias (mínimo=34 dias; máximo=310 dias) enquanto no subgrupo experimental a duração foi de 54,9 dias (mínimo=12 dias; máximo=142 dias). No subgrupo idoso a recuperação clínica no grupo controlo foi de 145,1 dias (mínimo=38 dias; máximo=365 dias) e no grupo experimental foi de 85 dias (mínimo=34 dias; máximo=194 dias).

Verificou-se uma relação significativa entre a idade e o nível de recuperação, a favor do subgrupo não idoso em ambos os grupos [grupo controlo $(p=0,010)$; grupo experimental $(p=0,000)]$. Os participantes do grupo experimental mostraram, em média e para qualquer idade, uma recuperação funcional mais rápida (70 dias versus 124 dias) quando comparados com os participantes do grupo controlo.

Este estudo apresenta como limitações o facto de ter sido realizado apenas em pacientes com PB severa (HB
$\mathrm{IV} \mathrm{eV}$ ) e por não discriminar em que fase da PB os doentes se encontravam. Atribuiu-se um NE 1.

\section{CONCLUSÃO}

A Canadian Medical Association ${ }^{3}$ não recomenda os exercícios de MFR na fase aguda, recomendando-os na fase crónica, mas apenas em doentes com fraqueza persistente. A National Guideline Clearinghouse $e^{7}$ não recomenda MFR, independentemente da fase da doença.

A RS de Teixeira e colaboradores ${ }^{4}$ conclui que não $^{2}$ existe evidência de elevada qualidade a favor de um benefício ou dano significativo de qualquer forma de MFR na PB. Existe evidência de baixa qualidade de que os exercícios de MFR melhoram a função facial, especialmente nos doentes com PB moderada ou na fase crónica, e de que reduzem o tempo total de recuperação e as sequelas na fase aguda.

A RS de Ferreira e colaboradores ${ }^{5}$ sugere que a MFR, associada a corticoterapia, parece ter um efeito positivo no grau e tempo de recuperação quando comparada com a corticoterapia isolada. Contudo, existe evidência de baixa qualidade dos ECAC, o que torna insuficiente para decidir a favor ou contra o tratamento combinado na recuperação da PB.

O estudo de coorte de Toffola e colaboradores ${ }^{2}$ conclui que o tratamento de MFR não é necessário nos doentes com neuropraxia, pois não existem sinais de desenervação, pelo que esses doentes recuperam espontaneamente. Nos doentes com axonotmese verifica-se uma melhoria sintomática, mas sem significado estatístico.

O ECAC de Monini e colaboradores, ${ }^{19}$ de agosto de 2016, conclui que quando a MFR é associada à corticoterapia nos casos de PB severa, os doentes apresentam uma maior probabilidade de recuperação mais rápida e completa do que aqueles submetidos apenas a tratamento com corticosteróides, com significado estatístico.

Outro ECAC, também de Monini e colaboradores, ${ }^{20}$ de dezembro de 2016, sugere a importância de combinar a MFR com o tratamento médico convencional para um melhor resultado na recuperação da PB severa em todos os grupos etários. Este tratamento combinado é particularmente importante nos doentes mais idosos e com grau mais severo de PB (HB V) que apresentam 
menor probabilidade de recuperação no mesmo tempo e grau que os doentes mais jovens.

Após a análise dos artigos conclui-se que os dados encontrados são limitados pelo tipo e validade dos estudos, os quais têm pouca qualidade e são muito heterogéneos. Dada a inconsistência dos resultados dos estudos quanto à utilização da MFR, tanto na fase aguda como crónica da PB, torna-se difícil concluir acerca dos seus benefícios. Na fase aguda são precisos mais ECAC para se poder determinar se a recuperação dos doentes é espontânea ou se é devida/acelerada por MFR. São ainda necessários mais ECAC de boa qualidade, em que o grupo controlo não inclua qualquer tipo de modalidade de MFR e em que as fases aguda e crónica da PB estejam bem definidas.

Não existe evidência para a recomendação ou para a não recomendação de MFR na fase aguda ou crónica, pelo que não se pode atribuir uma força de recomendação.

\section{REFERÊNCIAS BIBLIOGRÁFICAS}

1. Matos C. Paralisia facial periférica: o papel da medicina física e de reabilitação [Peripheral facial paralysis: the role of physical medicine and rehabilitation]. Acta Med Port. 2011;24(Suppl 4):907-14. Portuguese

2. Dalla Toffola E, Tinelli C, Lozza A, Bejor M, Pavese C, Degli Agosti I, et al. Choosing the best rehabilitation treatment for Bell's palsy. Eur J Phys Rehabil Med. 2012;48(4):635-42.

3. de Almeida JR, Guyatt GH, Sud S, Dorion J, Hill MD, Kolber MR, et al. Management of Bell palsy: clinical practice guideline. CMAJ. 2014; 186(12):917-22.

4. Teixeira LJ, Valbuza JS, Prado GF. Physical therapy for Bell's palsy (idiopathic facial paralysis). Cochrane Database Syst Rev. 2011;(12): CD006283.

5. Ferreira M, Marques EE, Duarte JA, Santos PC. Physical therapy with drug treatment in Bell palsy: a focused review. Am J Phys Med Rehabil. 2015; 94(4):331-40.

6. Murthy JM, Saxena AB. Bell's palsy: treatment guidelines. Ann Indian Acad Neurol. 2011;14(Suppl 1):S70-2.

7. Baugh RF, Basura GJ, Ishii LE, Schwartz SR, Drumheller CM, Burkholder $R$, et al. Clinical practice guideline: Bell's palsy. Otolaryngol Head Neck Surg. 2013;149(Suppl 3):S1-27.

8. Peitersen E. Bell's palsy: the spontaneous course of 2,500 peripheral facial nerve palsies of different etiologies. Acta Otolaryngol. 2002;549 Suppl:4-30.

9. Gronseth GS, Paduga R. Evidence-based guideline update: steroids and antivirals for Bell palsy: report of the Guideline Development Subcommittee of the American Academy of Neurology. Neurology. 2012;79(22):2209-13.
10. Braga R, Melo M. Como fazer uma revisão baseada na evidência [How to write an evidence based review]. Rev Port Clin Geral. 2009;25(6): 660-6. Portuguese

11. Ebell MH, Siwek J, Weiss BD, Woolf SH, Susman J, Ewigman B, et al. Strength of recommendation taxonomy (SORT): a patient-centered approach to grading evidence in the medical literature. Am Fam Physician. 2004;69(3):548-56.

12. Barbara M, Antonini G, Vestri A, Volpini L, Monini S. Role of Kabat physical rehabilitation in Bell's palsy: a randomized trial. Acta Otolaryngol. 2010;130(1):167-72.

13. Wen $C M$, Zhang BC. Effect of rehabilitation training at different degree in the treatment of idiopathic facial palsy: a randomized controlled comparison. Chin J Clin Rehabil. 2004;8(13):2446-7. Chinese

14. Beurskens $\mathrm{CH}$, Heymans PG. Positive effects of mime therapy on sequelae of facial paralysis: stiffness, lip mobility, and social and physical aspects of facial disability. Otol Neurotol. 2003;24(4):677-81.

15. Flores-Flores P, Medina-Zazueta R, Haro-García L. Tratamiento de la parálisis facial periférica idiopática: terapia física versus prednisona [Idiopathic peripheral facial paralysis treatment physic therapy versus prednisone]. Rev Med IMSS. 1998;36(3):217-21. Spanish

16. Nicastri M, Mancini P, De Seta D, Bertoli G, Prosperini L, Toni D, et al. Efficacy of early physical therapy in severe Bell's palsy: a randomized controlled trial. Neurorehabil Neural Repair. 2013;27(6):542-51.

17. Penteado TC, Testa JR, Antunes ML, Chevalier AM. Évaluation de la technique Chevalier pour la prévention des séquelles dans la paralysie faciale périphérique [Evaluation of the Chevalier method for the prevention of sequelae after peripheral facial nerve palsy]. Kinesither Rev. 2009;9(90):40-7. French

18. Alakram P, Puckree T. Effects of electrical stimulation on House-Brackmann scores in early Bell's palsy. Physiother Theory Pract. 2010;26(3): 160-6.

19. Monini S, lacolucci CM, Di Traglia M, Lazzarino Al, Barbara M. Role of Kabat rehabilitation in facial nerve palsy: a randomised study on severe cases of Bell's palsy. Acta Otorhinolaryngol Ital. 2016;36(4):282-8.

20. Monini S, Buffoni A, Romeo M, Di Traglia M, Filippi C, Atturo F, et al. Kabat rehabilitation for Bell's palsy in the elderly. Acta Otolaryngol. 2017;137(6):646-50.

\section{CONFLITO DE INTERESSES}

Os autores declaram não ter quaisquer conflitos de interesse.

\section{FINANCIAMENTO}

Os autores declaram não ter recebido subsídios ou bolsas para a elaboração do artigo.

\section{ENDEREÇO PARA CORRESPONDÊNCIA}

Sofia Oliveira Vale

E-mail: sofia.oliveira.vale@gmail.com

http://orcid.org/0000-0001-6940-0991

Recebido em 22-02-2017

Aceite para publicação em 07-02-2019 


\section{ABSTRACT}

PHYSICAL MEDICINE AND REHABILITATION IN THE TREATMENT OF BELL'S PALSY: WHAT'S THE EVIDENCE?

Objective: To review the scientific evidence of the impact of physical medicine and rehabilitation (PMR) in the recovery of acute and chronic Bell's Palsy recovery, in any degree of severity.

Data sources: National Guideline Clearinghouse, Guidelines Finder, Canadian Medical Association Infobase, Cochrane Library, DARE, and PubMed.

Methods: Search of clinical practice guidelines, meta-analyses, systematic reviews, and original studies, published in English, Spanish and Portuguese, published since 2006, using the MeSH terms 'peripheral facial paralysis', 'Bell's palsy', and 'rehabilitation'. The evidence level and the strength of recommendation of the studies were determined using the American Family Physician's Strength of Recommendation Taxonomy Scale.

Results: Among the 127 articles retrieved, seven met the inclusion criteria: two clinical guidelines, two systematic reviews, and three original studies. The Canadian Medical Association (2014) recommends the use of PMR in the chronic phase, but not on acute phase, whilst the National Guideline Clearinghouse (2013) does not recommend PMR, regardless of the phase. The systematic review by Ferreira et al. (2015) shows a greater motor recovery with PMR than with isolated pharmacological treatment, without phase discrimination. The systematic review by Teixeira et al. (2012) claims there is low-quality evidence showing that PMR is effective, both in the acute and chronic phases. The cohort study by Toffola et al. (2012) concludes that patients with axonotmesis in the acute phase show clinical improvement with PMR. The clinical randomised controlled trial by Monini et al. (2016 August) shows a greater and faster recovery with MFR than with pharmacological treatment alone in severe Bell's palsy. The clinical randomised controlled trial by Monini et al. (2016 December) suggests the importance of combining MFR with corticosteroid therapy for a better outcome in the recovery of severe BP in all age groups.

Discussion: The studies have low quality and are very heterogeneous. In the acute they do not allow to conclude whether patient recovery is spontaneous or due/accelerated by the use of PMR. There is no consistent evidence to recommend for or against PMR in the acute or chronic phases, therefore strength of recommendation cannot be attributed. More high-quality randomised controlled studies are needed.

Keywords: Peripheral facial paralysis; Bell's palsy; Rehabilitation. 